\title{
Stability structure of the GLR equation
}

\author{
Guillermo Gambini ${ }^{* \dagger}$ \\ University of Campinas \\ E-mail: guillerivgambegmail.com \\ Giorgio Torrieri * \\ University of Campinas \\ E-mail: torrierieifi.unicamp.br
}

We show that the GLR equation develops unstable modes when the parton distribution function is generalized to depend on azimuthal angle. This generally means the saturation scale $Q_{s}$ acquires an azimuthal dependence. We argue that this process is a plausible alternative explanation for the origin of elliptic flow, one that naturally respects the scaling experimentally observed.

XXIV International Workshop on Deep-Inelastic Scattering and Related Subjects

11-15 April, 2016

DESY Hamburg, Germany

\footnotetext{
*Speaker.

${ }^{\dagger}$ GG is supported by National Council for Scientific and Technological Development - CNPq

$\ddagger_{\text {GT }}$ acknowledges support from FAPESP proc. 2014/13120-7 and CNPq bolsa de produtividade 301996/2014-8.
} 
Hydrodynamics is the most favored explanation for the anisotropic flow observed in nucleusnucleus, and now proton-nucleus and even proton-proton collisions (for a review see [1]). However, several phenomenological puzzles (see [2] and references therein for a review) have accumulated challenging hydrodynamics as the origin of $v_{n}$. For instance, the near independence of $v_{2}$, and particularly, $v_{3}$, on system size, i.e. $p A, d A$, and even $p p$ collisions give you, scaled for geometry, the same azimuthal coefficients as $A A$ collisions [3,4]. As a related item, the near independence of $v_{2}\left(p_{T}\right)$ on energy $[6,8]$ and rapidity [7, 9]. Also, the photon and dielectron $v_{2}$ is comparable to the hadron $v_{2}$. Usual explanations aim to provide an enhancement of this $v_{n}$ but never to explain the equality [10].

All of these, summarized in [2], point to an origin of $v_{2}$ which is not hydrodynamic but rather based on initial conditions, since it resembles the scaling of structure functions $f\left(x, Q^{2}\right)$ and fragmentation functions $D_{q \rightarrow i}\left(z, Q^{2}\right)$ [11]. Hence, if we could just assume that either the structure function acquired an azimuthal dependence having the usual Bjorken scaling (strong and fundamentally non-perturbative dependence on $x$ ), logarithmically suppressed dependence on $Q^{2}$, and this azimuthal dependence generated most of $v_{2}$, all issues described in this introduction would be naturally resolved.

Such a "simple" suggestion of course is superficially extremely implausible: QCD has azimuthal symmetry, and the parton structure and fragmentation functions are based on factorization at high energy scales. Thus, they are "universal" and should not depend on relative angles, even if the target and/or projectile are spatially extended. Yet, ways compatible with $Q C D$ to incorporate azimuthal dependence of structure functions do exist. In fact, since the distributions functions depend on the probe used to measure them beyond tree level ("run"), a dipole colliding off-center with an extended object will measure azimuthally asymmetric parton distribution functions to precision to one-loop. Since the impact parameter $b \sim \mathscr{O}(1-10) \mathrm{fm}$, these corrections are expected to be tiny $\sim \alpha_{s}^{2} / b$, hence, provided evolution is linear, their contribution to the observed $v_{n}$ is undetectable.

In this work, however, we investigate whether azimuthal symmetry is spontaneously broken by the same processes that are thought to lie at the core of saturation physics. If it is, the above anisotropies are indeed tiny at high rapidities but could be amplified at mid-rapidity, in the same way as cylindrical boundary conditions in a flow of water from a faucet lead to an azimuthally asymmetric turbulent flow when density and flow velocity are high enough.

\section{Stability of the GLR equation}

The GLR equation is the leading-order non-linear correction to the BFKL equation, which is thought to be valid in the high density limit, when the transverse wavefunctions of the partons overlap.

We shall consider the GLR equation in its purely differential (Mueller-Qiu) limit. In terms of the structure function $G$, Bjorken $x$, strong coupling constant $\alpha_{s}$, and transverse diameter of the nucleus $S_{\perp}$, it is

$$
\frac{\partial^{2} x G\left(x, Q^{2}\right)}{\partial \ln (1 / x) \partial \ln \left(Q^{2} / \Lambda^{2}\right)}=\frac{\alpha_{s} N_{c}}{\pi} x G\left(x, Q^{2}\right)-\frac{\alpha_{s}^{2} \pi}{S_{\perp}} \frac{1}{Q^{2}}\left[x G\left(x, Q^{2}\right)\right]^{2} .
$$


Assuming azimuthal symmetry, everything depends on $Q$ rather than $Q_{x}$ and $Q_{y}$, or equivalently in Eq. $1.1 G(x, k, \theta)=G(x, k, \theta+\phi)$ and the angle can be integrated out. Neglecting angular effects (which are assumed to be suppressed by factors of $Q$ ), the most trivial modification one can make is to relax this approximation. It is easy to see that this modifies Eq. 1.1 by a relatively simple substitution

$$
\frac{1}{\Lambda^{2}}\left(\frac{\partial}{\partial Q}+\frac{1}{Q} \frac{\partial}{\partial \theta}\right) \frac{\partial x Q^{2} G\left(x, Q^{2}\right)}{\partial \ln (1 / x)}=\frac{\alpha_{s} N_{c}}{\pi} x G\left(x, Q^{2}\right)-\frac{\alpha_{s}^{2} \pi}{S_{\perp}} \frac{1}{Q^{2}}\left[x G\left(x, Q^{2}\right)\right]^{2},
$$

We intend to perturb the solutions of Eq.1.1, i.e. $G_{0}\left(x, Q^{2}\right)$, the following way

$$
G\left(x, Q^{2}, \theta\right)=G_{0}\left(x, Q^{2}\right)\left(1+\sum_{n} u_{n}\left(x, Q^{2}\right) \cos \left(n \theta+\beta_{n}\right)\right),
$$

where the background $G_{0}\left(x, Q^{2}\right)$ is modeled as the (azimuthally symmetric) saturation scenario $[12,13]$, the solution of the azimuthally symmetric non-linear equation, which is a transcendental function approximately equal to

$$
G_{0}\left(x, Q^{2}\right)=\frac{x^{2 \lambda}}{2 \alpha_{s}^{4}}\left[(1-\tanh (\xi))+\frac{Q_{s}^{2}(x)}{Q^{2}}(1+\tanh (\xi))\right],
$$

with $Q_{s}(x)=\alpha_{s}^{2} \Lambda_{Q C D} x^{-\lambda}, \xi=\frac{Q-Q_{s}(x)}{\zeta}$ and $\zeta \sim \frac{\alpha_{s} \Lambda_{Q C D}}{N_{c}}$, so that $G_{0}(x, Q)$ has the approximate step function form determined by requiring the two sides of Eq. 1.1 to balance

$$
Q_{s}^{2}(x)=\frac{\alpha_{s} \pi^{2}}{S_{\perp} N_{c}} x G_{s}\left(x, Q_{s}(x)\right) \rightarrow Q_{s}(x) \sim x^{2 \lambda+1}
$$

It is of special interest for us to study parton distribution functions when the probe energy is small compared to the saturation scale; i.e. $Q / Q_{s}(x) \ll 1$. In this limit we obtain

$$
\begin{gathered}
(2 \lambda+1) \frac{Q}{2} \frac{\partial u_{n}\left(x, Q^{2}\right)}{\partial Q}+\frac{Q}{2} x \frac{\partial^{2} u_{n}\left(x, Q^{2}\right)}{\partial Q \partial x}=\left[\frac{\alpha_{s} N_{c}}{\pi}+\frac{N_{c} \pi}{C_{F} S_{\perp} \alpha_{s}^{2}} \frac{x^{2 \lambda+1}}{Q^{2}}\right] u_{n}\left(x, Q^{2}\right)+\delta u_{n}(x, Q) \\
\delta u_{n}(x, Q)=\frac{N_{c} \pi}{2 C_{F} S_{\perp} \alpha_{s}^{2}} \frac{x^{2 \lambda+1}}{Q^{2}}-\left[\frac{1}{2} \sum_{k}^{n-1} u_{k}\left(x, Q^{2}\right) u_{n-k}\left(x, Q^{2}\right) \cos \left(\beta_{n}-\beta_{k}-\beta_{n-k}\right)\right. \\
\left.+\sum_{k} u_{k}\left(x, Q^{2}\right) u_{n+k}\left(x, Q^{2}\right) \cos \left(\beta_{n}+\beta_{k}-\beta_{n+k}\right)\right] .
\end{gathered}
$$

Assuming $\delta u_{n}$ is small (a Taylor expansion can go beyond this approximation), one can study the behavior of linearized instabilities (where the instability interacts with the azimuthally symmetric "saturating" component) relatively easily.

A thought needs to go into boundary conditions. The running scenario we described earlier would assume that the perturbation starts at a non-zero $x$ and grows with $x \rightarrow 0$. An ansatz appropriate for these boundary conditions is $u\left(x, Q^{2}\right)=x^{p} f(t)$ with $t=B x^{C} / Q^{2}=z^{2} / 2 D, B=\pi^{2} / C_{F} S_{\perp} \alpha_{s}^{3}$, 
$C=2 \lambda+1$, and $D=2 \alpha_{s} N_{c} /(2 \lambda+1) \pi$. So, we get a Bessel's equation with pure imaginary index (order) $i v$ for $f$

$$
f^{\prime \prime}+\frac{1}{z} f^{\prime}+\left(1+\frac{v^{2}}{z^{2}}\right) f=0,
$$

when $p=-C$ and $v=\sqrt{2 D}$. The solution of this equation is inspired by Bessel functions of the first kind [14]

$$
f_{v}(z)=A(z) \cos (v \ln z)+B(z) \sin (v \ln z) \quad, \quad \begin{aligned}
& A(z) \\
& B(z)
\end{aligned}=\sum_{n=0}^{\infty} A_{2 n} B_{2 n}\left(\frac{z}{2}\right)^{2 n}
$$

with a recursive definition

$$
A_{2 n}=-\frac{n A_{2 n-2}-v B_{2 n-2}}{n\left(n^{2}+v^{2}\right)}, B_{2 n}=-\frac{v A_{2 n-2}+n B_{2 n-2}}{n\left(n^{2}+v^{2}\right)}
$$

for $n \geq 1$ and $A_{0}, B_{0}$ known. Finally, our solution reads

$$
u\left(x, Q^{2}\right)=x^{-(2 \lambda+1)} f_{v}(z), z=\sqrt{\left(\frac{4 \pi N_{c}}{(2 \lambda+1) C_{F} \alpha_{s}^{2}}\right) \frac{1}{S_{\perp}} \frac{x^{2 \lambda+1}}{Q^{2}}}, \quad v=\left(\frac{4 \alpha_{s} N_{c}}{\pi(2 \lambda+1)}\right)^{1 / 2}
$$

We start evolution at a critical moderately high $x$ to fix the parameters characterizing the theory ( $A_{0}, B_{0}$ for the Bessel function) to reproduce $u_{2} / \varepsilon \sim 10^{-2}$, in line with expectation from semiperturbative higher twist processes. We then go lower in $x$ to analyze the behavior of $u_{2}, v_{2}$ as one gets closer to mid-rapidity. As can be seen in Fig. 1 (left panel) the instability indeed grows extremely rapidly from a broad value of Bjorken $x$. In fact, very quickly it becomes so large that the linearization ansatz we used becomes inapplicable.

As a phenomenological fix to deal with this issue, we shall assume corrections beyond the leading order "saturate" $u_{2}$. An ad-hoc but consistent way to do this is replacing $u_{2}(x, Q) \rightarrow$ $u_{2}^{\max } \tanh \left(\frac{u_{2}(x, Q)}{u_{2}^{\max }}\right)$, so that at low $u_{2}$ this recovers the linearized equations but never goes above the parameter $u_{2}^{\max }$. Physically, this will be the scale where higher order corrections, assumed here to essentially cutoff $u_{2}$ growth, take over. The price for this adjustment is that we lose predictivity of $u_{2}$ at a given $Q, x$.

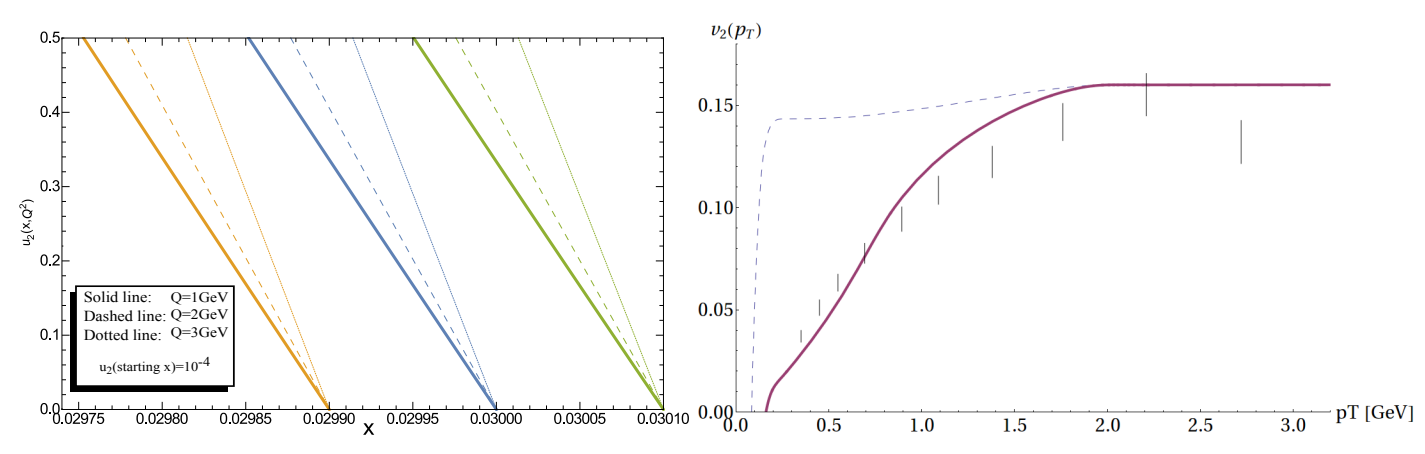

Figure 1: Left panel: $u_{2}$ as a function of $x, Q$ when started as a small value at a "moderate" $x$ (where processes such as higher twist can occur) and evolved to $x \rightarrow 0$. Right panel: a comparison of the data with a stabilized perturbation, converted into hadrons via $k_{T}$ factorization and limiting fragmentation 
To convert these asymmetric distribution functions to something that can be related to experimental measurement, we would need to perform microscopic quark-and gluon scattering as well as use fragmentation functions. For a rough estimate we use the $k_{T}$ factorization ansatz $[11,13]$

$$
\left.\frac{d N}{d p_{T} d y} \sim \frac{1}{p_{T}^{2}} \int \delta\left(x_{A}+x_{B}-e^{-y}\right) k d k d \theta f_{A}\left(x_{A}, k, \theta\right) f_{B}\left(x_{B}, p_{T}-k, \pi+\phi-\theta\right)\right|_{x_{A, B}=\frac{p_{T} e^{ \pm y}}{\sqrt{s}}} .
$$

Motivated by Eq. 1.11 we set $u_{2}^{\max }$ at a value of 0.5 , where its contribution to hadron production becomes comparable to the unprerturbed, saturation value. We note that the experimental $v_{2}$ is curiously quantitatively similar to this limit.

Together with quark-hadron duality, $k_{T}$ factorization can be used to provide a calculation for $v_{2}$. The shifting of momentum due to fragmentation from parton $p_{T}$ to hadron $p_{T h}$, necessary to provide the right limit for $d N / d p_{T}$, can be accomplished by updating Eq. 1.11 using

$\frac{d N_{h}}{d p_{T h} d y_{h}}=\left.\int_{0}^{1} d z \int_{\Lambda_{Q C D}}^{Q_{s}} d p_{T} \delta\left(z-\frac{p_{T h}}{p_{T}}\right) z D(z, p T) \frac{d N}{d p_{T} d y}\right|_{p_{T}}=\left.\int_{p_{T h} / Q_{s}}^{1} d z z \int_{\Lambda_{Q C D} / z}^{Q_{s}} D(z, p T) \frac{d N}{d p_{T} d y}\right|_{p_{T}}$.

We do this with a Schwinger function-inspired Gaussian fragmentation function, $D(z, p T) \sim e^{-z^{2}}$. Note that the integration limits are for the parton to have momentum larger than $\Lambda_{Q C D}$ and smaller than $Q_{s}$. The results, to be interpreted as a rough estimate, are shown in the right panel of Fig. 1.

In conclusion, we have discussed the phenomenological scaling of azimuthal anisotropy coefficients in hadronic collisions, arguing that this suggests an initial state origin compatible with "Bjorken" type scaling phenomenology. We have also noted that saturation dynamics corresponds also to a regime where azimuthal instabilities, in the full $(2+1)$ evolution equations, could acquire growing modes. We have used the azimuthally asymmetric GLR equation as a laboratory to test these growing modes, and found intriguing hints that they are indeed possible and have some qualitative features required for modeling $v_{n}$. This suggests that this model should be developed further in order to connect it with data at a quantitative level, including the full integro-differential equation, realistic particle production and fragmentation, etc. This is something that we plan to do in a subsequent work.

\section{References}

[1] R. D. de Souza, T. Koide and T. Kodama, arXiv:1506.03863 [nucl-th].

[2] G. Gambini and G. Torrieri, arXiv:1606.07865 [nucl-th].

[3] S. Chatrchyan et al. [CMS Collaboration], Phys. Lett. B 718, 795 (2013)

[4] A. Adare et al. [PHENIX Collaboration], Phys. Rev. Lett. 111, no. 21, 212301 (2013) doi:10.1103/PhysRevLett.111.212301 [arXiv:1303.1794 [nucl-ex]].

[5] V. Khachatryan et al. [CMS Collaboration], arXiv:1606.06198 [nucl-ex].

[6] Phys. Rev. C 86, 054908 (2012) doi:10.1103/PhysRevC.86.054908 [arXiv:1206.5528 [nucl-ex]].

[7] BRAHMS collaboration, Nucl. Phys. A 830 43C (2009)

[8] CMS collaboration, PRL 109022301 (2012) 
[9] PHOBOS collaboration, Nucl. Phys. A830 (2009)

[10] A. Adare et al. [PHENIX Collaboration], Phys. Rev. Lett. 109, 122302 (2012) [arXiv:1105.4126 [nucl-ex]].

[11] J.Ellis,W.J. Stirling, B.R. Webber, QCD and collider Physics, Cambridge monographs on particle physics, nuclear physics and cosmology

[12] F. Gelis, E. Iancu, J. Jalilian-Marian and R. Venugopalan, Ann. Rev. Nucl. Part. Sci. 60, 463 (2010) [arXiv:1002.0333 [hep-ph]].

[13] Y. V. Kovchegov and E. Levin, "Quantum chromodynamics at high energy”, CUP

[14] A. A. Matyshev and E. Fohtung, arXiv:0910.0365[math-ph] 